\title{
NECESSARY AND SUFFICIENT CONDITIONS FOR RECURRENCE AND TRANSIENCE OF MARKOV CHAINS, IN TERMS OF INEQUALITIES
}

\author{
JEAN-FRANÇOIS MERTENS, Université de Louvain \\ ESTER SAMUEL-CAHN, Hebrew University of Jerusalem \\ SHMUEL ZAMIR, Hebrew University of Jerusalem
}

\begin{abstract}
For an aperiodic, irreducible Markov chain with the non-negative integers as state space it is shown that the existence of a solution to $\sum_{j=0}^{\infty} p_{i j} y_{i} \leqq y_{i} ; i \geqq N>0$ in which $y_{i} \rightarrow \infty$ is necessary and sufficient for recurrence, and the existence of a bounded solution to the same inequalities, with $y_{k}<y_{0}, \cdots, y_{N-1}$ for some $k \geqq N$, is necessary and sufficient for transience.

RECURRENCE; TRANSIENCE
\end{abstract}

Consider a Markov chain with the non-negative integers as its state space and transition matrix $P=\left(p_{i j}\right)$, and for simplicity assume that the chain is irreducible and aperiodic.

Theorem 1. A necessary and sufficient condition that the Markov chain be recurrent is that there exists a solution to the system of inequalities

(1) $\sum_{j=0}^{\infty} p_{i j} y_{j} \leqq y_{i} ; i \geqq N, \quad$ for some integer $N>0$, such that $\lim y_{i}=\infty$.

Sufficiency of the condition of Theorem 1 is well known. For $N=1$ this is Theorem 5 in Foster (1953). Foster states that 'the condition would appear necessary only under certain additional assumptions'. For $N \geqq 1$ the sufficiency is proved in Pakes (1969), Theorem 3. For the sake of completeness, and in its own interest, we include a different proof of the sufficiency.

Proof of sufficiency. Let $\boldsymbol{y}$ be a solution of (1) such that $\lim y_{i}=\infty$. By possibly adding a constant to all components of $y$ we may without loss of generality assume $y_{i} \geqq 0$. Define $y^{*}$ by $y_{i}^{*}=y_{i}$ for $i \geqq N, y_{i}^{*}=0$ for $i<N$ and let $P^{*}$ be obtained from $P$ by setting $p_{i j}^{*}=p_{i j}$ for $i \geqq N$, and $p_{i i}^{*}=1$ for $i<N$. Then $P^{*}$ and $y^{*}$ satisfy $\Sigma_{j=0}^{\infty} p_{i j}^{*} y_{j}^{*} \leqq y_{i}^{*}, i \geqq 0$. Let $Z_{0}=N$ and let $Z_{n} ; n \geqq 1$, be the state at time $n$ of the Markov chain governed by $P^{*}$. Note that the original chain

Received 14 October 1977; revision received 26 January 1978. 
is recurrent if and only if $P\left[\lim _{n \rightarrow \infty} Z_{n} \in\{0, \cdots,(N-1)\}\right]=1$, i.e. iff $P\left[\lim _{n \rightarrow \infty} Z_{n}=\infty\right]=0$. Let $X_{n}=y_{Z_{n}}^{*}$, and denote by $\mathscr{F}_{n}$ the $\sigma$-field generated by $\left\{Z_{i} ; i \leqq n\right\}$. Then $\left\{X_{n}, \mathscr{F}_{n}, n \geqq 0\right\}$ is an unbounded supermartingale for which $\sup _{n} E X_{n}^{-}=0$, and by the martingale convergence theorem (see e.g. Chow, Robbins and Siegmund (1971), p. 16) $\lim _{n \rightarrow \infty} X_{n}=X_{\infty}$ exists a.s. and satisfies $E X_{\infty}<\infty$. Now if $P\left[\lim _{n \rightarrow \infty} Z_{n}=\infty\right]>0$ we would have $P\left[X_{\infty}=\infty\right]>0$ and $E X_{\infty}=\infty$. Thus $P\left[\lim _{n \rightarrow \infty} Z_{n}=\infty\right]=0$.

Proof of necessity. Assume $P$ to be recurrent, and let $\tilde{P}$ be obtained from $P$ by setting $\tilde{p}_{i j}=p_{i j}$ for $i \geqq 1$, and $\tilde{p}_{00}=1$. We shall construct a sequence $\boldsymbol{y}$ satisfying (1), with $y_{i} \rightarrow \infty$. Let $f_{i}(n)$ be the probability that the Markov chain starting at $i$ and governed by $\tilde{P}$ ever enters the set of states $\{n, n+1, \cdots\}$. Thus $f_{0}(n)=0$ for all $n>0$ and $f_{i}(n)=1$ for $i \geqq n$. Note that since the original chain is recurrent, the new chain, starting at any state $i$, will eventually be absorbed in state 0 , with probability 1 . Thus

$$
\lim _{n \rightarrow \infty} f_{i}(n)=0 \text { for all } i \geqq 0 .
$$

We shall now define an increasing sequence $\left\{n_{k}\right\}$ to satisfy the requirements: $f_{1}\left(n_{1}\right)<\frac{1}{2} ; f_{1}\left(n_{2}\right)<\frac{1}{4}, f_{2}\left(n_{2}\right)<\frac{1}{4}$, and for any $k$ choose $n_{k}$ to satisfy $f_{i}\left(n_{k}\right)<2^{-k}$ for all $i \leqq k$. By (2) this is clearly possible. Note that for any fixed $n$ the sequence $\left\{f_{i}(n)\right\}$ satisfies (1) with $N=1$. Define $y$ by $y_{i}=\sum_{k=1}^{\infty} f_{i}\left(n_{k}\right)$. Since $f_{i}\left(n_{k}\right)<2^{-k}$ for all $k \geqq i$ it follows that $y_{i}<\infty$, and $\boldsymbol{y}$ satisfies (1) with $N=1$. Furthermore, for any fixed $k f_{i}\left(n_{k}\right) \rightarrow 1$ as $i \rightarrow \infty$, and hence $\lim y_{i}=\infty$ by Fatou's lemma.

Remark. One may wonder whether the sufficient condition could be weakened to say that (1) should hold with $y$ such that lim sup $y_{i}=\infty$. The answer to this question is in the negative, as seen by the following example. Let $\{0,2,4 \cdots\}$ be the state space of an aperiodic transient irreducible chain with transition matrix $P$. Then by a well-known theorem (see e.g. Foster (1953), Theorem 4, or Feller (1968), p. 402), there exist non-constant $0 \leqq y_{2 i} \leqq 1$ solving $\sum_{j=0}^{\infty} P_{2 i, 2 j} y_{2 j}=y_{2 i}$ for all $i \geqq 1$.

We shall now add states $\{1,3, \cdots\}$, and define the transition matrix $\tilde{P}$ of the new chain by

$$
\begin{aligned}
& \tilde{p}_{2 i, 2 j}=p_{2 i, 2 j} \text { for all } i \geqq 1 . \\
& \tilde{p}_{0,2 j}=p_{0,2 j} / 2, j \geqq 0, \text { and } \tilde{p}_{0,1}=\frac{1}{2} . \\
& \tilde{p}_{2 i+1,2 i+2}=\frac{1}{2}=\tilde{p}_{2 i+1,2 i+3} ; i \geqq 0 .
\end{aligned}
$$

It is clear that $\tilde{P}$ represents an aperiodic irreducible chain. Moreover, the chain is transient, since it will, with probability one, end up among the positive even states, for which we have not changed the transition probabilities and hence the probability of returning to 0 is less than 1 . Now let $\tilde{y}$ be defined by $\tilde{y}_{2 i}=y_{2 i} i \geqq 0$, 
and $\tilde{y}_{2 i+1}=i+2$. Then clearly $\sum_{j=0}^{\infty} \tilde{p}_{i j} \tilde{y}_{j} \leqq \tilde{y}_{i}$ holds for all $i \geqq 1$, since for $i=2 k$, $k>0$, these inequalities hold with equality and for $i=2 k+1$ we have

$$
\sum_{j=0}^{\infty} \tilde{p}_{2 k+1, j} \tilde{y}_{j}=\frac{1}{2} \tilde{y}_{2 k+2}+\frac{1}{2} \tilde{y}_{2 k+3} \leqq \frac{1}{2}+\frac{1}{2}(k+3)=\frac{1}{2} k+2 \leqq k+2 \text { for all } k \geqq 0 .
$$

Thus lim sup $\tilde{y}_{i}=\infty$, but the chain is transient.

Theorem 2. A necessary and sufficient condition that the Markov chain be transient is that there exists a bounded solution to the system of inequalities

$$
\sum_{j=0}^{\infty} p_{i j} y_{j} \leqq y_{i}, \quad i \geqq N
$$

for some integer $N>0$, and for some $k \geqq N, y_{k}<y_{0}, \cdots, y_{N-1}$.

Necessity follows from Theorem 4 of Foster (1953). For $N=1$ the theorem is Theorem 6 in Foster (1953). For $N>1$ sufficiency is proved by Harris and Marlin (1972), Theorem 1, under the additional conditions that $y_{i}>0$ for $i<N$, and $y_{i} \rightarrow 0$. Their proof can be modified slightly to hold under our weaker conditions. A proof along the lines of proof of sufficiency in our previous theorem is as follows.

Let $\boldsymbol{y}, P^{*}$ and $\boldsymbol{y}^{*}$ be defined as in the proof of the sufficiency in Theorem 1 . Let $Z_{0}=k$ and let $Z_{n} ; n \geqq 1$, be the state at time $n$ of the chain governed by $P^{*}$. Let $X_{n}=y_{Z_{n}}^{*}$. Then $\left\{X_{n}, \mathscr{F}_{n}, n \geqq 0\right\}$ is a bounded supermartingale, and $\lim X_{n}=$ $X_{\infty}$ exists a.s. and satisfies $E X_{\infty} \leqq E X_{0}=y_{k}<y_{0}, \cdots, y_{N-1}$. Thus $P\left[\lim _{n \rightarrow \infty} Z_{n} \in\{0, \cdots,(N-1)\}\right]<1$ and $P$ is transient.

\section{Remarks}

(a) The requirement in (3) that $y_{k}<y_{0}, \cdots, y_{N-1}$ for some $k$, cannot be disposed of, since $\boldsymbol{y}=(0,1,1, \cdots)$ is bounded and satisfies the set of inequalities in (3) for any transition matrix $P$.

(b) Equivalent statements of the theorems are obtained by letting the summation in (1) and (3) start from $j=1$, (by setting $y_{0}=0$ ) and making the relevant changes.

\section{Acknowledgement}

The authors want to thank the referee for his constructive remarks on an earlier version of this note.

\section{References}

Chow, Y. S., Robbins, H. And SiEgmund, D. (1971) Great Expectations: The Theory of Optimal Stopping. Houghton Mifflin, Boston. 
Feller, W. (1968) An Introduction to Probability Theory and its Applications. Vol. I, 3rd edn. Wiley, New York.

Foster, F. G. (1953) On the stochastic matrices associated with certain queuing processes. Ann. Math. Statist. 24, 355-360.

Harris, C. M. And Marlin, P. G. (1972) A note on feedback queues with bulk service. J. Assoc. Comput. Mach. 19, 727-733.

PAKes, A. G. (1969) Some conditions for ergodicity and recurrence of Markov chains. Opns Res. 17, 1058-1061. 